\title{
Improving the teaching of science through discipline-based education research: An example from physics
}

\author{
Lillian C. McDermott \\ Department of Physics, University of Washington, Seattle, Washington, U.S.A \\ For correspondence: 1cmcd@phys.washington.edu
}

\begin{abstract}
Research on the learning and teaching of science is an important field for scholarly inquiry by faculty in science departments. Such research has proved to be an efficient means for improving the effectiveness of instruction in physics. A basic topic in introductory physics is used to illustrate how discipline-based education research has helped identify certain conceptual and reasoning difficulties that are common among university students and pre-university teachers. The results have been used to guide the design of instruction that has brought about a significant improvement in learning. The type of research illustrated requires a deep knowledge of physics and ready accessibility to students as they study that subject. Both of these conditions are usually present only in physics departments, not in departments in which the primary focus is on educational theory and methodology. Although the context of this paper is physics, analogies can readily be made to other sciences.
\end{abstract}

\section{Introduction}

The Physics Education Group at the University of Washington (UW) has demonstrated that the learning and teaching of physics can be investigated in a scientifically rigorous manner. We have drawn on the results to guide the development of two sets of research-based curricula, which are widely distributed. Both are also research-validated, in that they have led to documented improvement in student learning. Our experience suggests that discipline-based education research can be an efficient means for achieving cumulative improvement in the effectiveness of instruction. Our emphasis has been on university students and on elementary and secondary school teachers, but we have evidence that this approach is also useful at other levels of education.

Many university science faculty view teaching solely as an art and maintain that it can never be a science. They seem to regard discipline-based education research only as a means for instructors to improve their own lectures. The goal of this paper is to demonstrate that this field of research can be an efficient means for achieving widespread and ongoing cumulative improvement in the effectiveness of instruction. The context is physics but analogies can readily be made to other sciences. In the discussion that follows, the examples used as illustrations are from a basic topic in physics that most science faculty have studied at some time during their secondary or university education.

\section{Brief History}

The Physics Education Group at the University of Washington consists of faculty, postdoctoral research associates, pre-university teachers, and graduate students. It is an integral part of the Physics Department. The group has welcomed many visiting faculty and graduate students, some of whom have joined us for an extended period of time. We conduct a coordinated program of research on student understanding, curriculum development, and instruction. Our graduate students earn a Ph.D. in physics (not in physics education). They meet all of the same requirements as all other graduate 
students in the Department. Since 1979, 22 have earned a Ph.D. through their research with our group.

Our group is unusual among physicists who conduct research in physics education in the U.S. because our involvement grew out of our work with prospective (preservice) and practicing (inservice) teachers. For more than 35 years, we have been teaching special courses in physics for preservice elementary and secondary school teachers during the academic year and conducting intensive six-week Summer Institutes for inservice teachers. (These have been supported by the U.S. National Science Foundation through a series of competitive grants.) We also offer a special weekly course during the entire academic year for all teachers who have participated in our Summer Institutes or in our academic-year courses for teachers. The development of our curriculum for the preparation of teachers has taken place in all of these instructional environments. In 1991 our research and curriculum development expanded to a major involvement in the introductory calculus-based physics course. We have since extended our research and development of curriculum to include more advanced physics courses.

Our two published curricula are Physics by Inquiry (PbI) and Tutorials in Introductory Physics (McDermott, 1996; McDermott and Shaffer, 2002). The first is a self-contained, laboratory-centered curriculum especially appropriate for the preparation of elementary and secondary school teachers that has also been used successfully with other students; the second is a supplementary curriculum consisting of tutorials that are intended for use with university students in conjunction with any standard introductory text. Both have been developed through an iterative cycle of research, curriculum development, and instruction. PbI has been translated into Greek, Polish, and Spanish. There are German, Greek, Korean, and Spanish translations of the tutorials.

The results from our research and curriculum development have been published in many articles. The context for the present discussion is geometrical optics. The reason for this choice is that almost everyone who has ever studied physics has had some experience in using a ray model to account for the reflection and refraction of light. This paper draws on papers in AJP (Goldberg and McDermott, 1987) and in Optics and Photonics News (Heron and McDermott, 1998).

\section{Research perspective}

The focus of research by the Physics Education Group is on the student as a learner, rather than on the instructor as a teacher. We try to identify specific difficulties that students encounter in the study of various topics. Several different populations were included in the research discussed in this paper: university students in introductory physics courses, elementary and secondary school teachers of physics and physical science, and graduate students whose research interests covered a wide range of subfields in experimental and theoretical physics.

The type of discipline-based education research that is conducted by our group is motivated by our interest in determining student understanding of physics content. Although the emphasis in this paper is on students in introductory university physics courses, we have found that elementary and secondary school teachers, and even physics graduate students, often have similar conceptual and reasoning difficulties. Our analysis of these findings guides our design of instructional strategies to address specific difficulties. Ongoing assessment is an integral part of this process. We have found that both the difficulties that we have identified and the instructional strategies that we have developed are often generalizable beyond a particular course, instructor, or institution. We work toward cumulative improvement in student learning through an iterative process of research, curriculum development, and instruction. We assess the effectiveness of our instructional materials through rigorous pre- and post-testing, document our methods so that they can be replicated, and 
report the results at meetings and in peer-reviewed papers. These procedures are characteristic of an empirical applied science.

Although experienced instructors know that there is a gap between what is said in lectures and what students learn, most do not recognize how large the gap can be. We have examined student understanding of many topics and have found that on certain types of qualitative questions, student performance is essentially the same: before and after instruction by lecture, laboratory, and textbook; irrespective of the level of mathematics; whether or not there is a standard laboratory; whether or not demonstrations are used; whether classes are large or small; and regardless of the proficiency of the lecturer. There is ample evidence that teaching by telling is ineffective for most students. Meaningful learning requires active mental engagement. The challenge, especially in large courses, is achieving the necessary degree of intellectual involvement. Much of our research has been directed toward responding to this challenge.

We use two general research methods: individual demonstration interviews (which allow deep probing into the nature of student difficulties) and written tests (which yield information on prevalence). Both methods provide a means for assessing student learning before and after instruction. Observations and interactions with students in the classroom lead to additional insights into how students learn best. The results inform the design of carefully sequenced questions in our curricula that are intended to address specific difficulties and to guide students through the process of constructing and applying a coherent conceptual framework in a variety of topics in physics.

\section{Instruction through guided inquiry}

Both Physics by Inquiry (PbI) and Tutorials in Introductory Physics engage students actively in the process of learning physics. In both curricula, teaching is by questioning, rather than by telling. The purpose of the questions that are asked is to force students to do the required reasoning for themselves. The ability to do, and to be able to articulate, the reasoning involved in applying a concept is emphasized. Our characterization of this mode of instruction is guided inquiry.

$\mathrm{PbI}$ is much more thorough than the tutorials. It is particularly important that teachers develop a deeper understanding of the material than most introductory physics students. There are no lectures in our special courses for teachers. We began developing $\mathrm{PbI}$ more than 30 years ago expressly for the preparation of elementary and secondary school teachers to teach physics and physical science. We have often found that elementary school teachers who have worked through $\mathrm{PbI}$ perform better on certain kinds of questions than physics and engineering students in introductory university physics courses. The tutorials are a "band-aid" approach, which is a compromise that satisfies the demands of a standard introductory physics course in which there is a great deal of coverage in very little time. Students are expected to work through all or most of a tutorial in 50 minutes. The same topic in $\mathrm{PbI}$ takes students several hours to complete.

Assessment of the effectiveness of both curricula is through pre-testing and post-testing that take place on a continuous basis. To ensure applicability beyond our own university, our instructional materials are tested at pilot sites that range from large universities to small colleges

\section{Identification of student difficulties with geometrical optics.}

In teaching geometrical optics, most instructors begin with the premise that students taking introductory physics at a university have a functional understanding of the rectilinear propagation of light. Most students can readily articulate that light travels in a straight line. They also seem to accept the idea that light rays travel outward from every point on an object and are converged by a convex lens to a corresponding point on an image. Moreover, almost all can use the thin lens formula $(1 / \mathrm{s}+$ 
$1 / \mathrm{s}^{\prime}=1 / \mathrm{f}$ ) to find the distance of an image from a lens, given the object distance and focal length. The two examples that follow show what many university students and pre-university teachers often cannot do.

Research Task: Real image formed by a converging lens While Fred Goldberg (now at San Diego State) was a visitor with our group during the 1980s, we examined how introductory students interpret the real image formed by a converging lens (Goldberg and McDermott, 1987). Fred conducted about 20 individual demonstration interviews with volunteers from the top half of their respective classes. Each interview lasted from 45 to 60 minutes. The questions posed were based on the apparatus shown in Fig. 1, which consists of a clear, brightly lit bulb, a converging lens, and the inverted real image of the filament of the bulb on a screen. The students were asked if anything would change on the screen if certain specified changes were made to the apparatus.

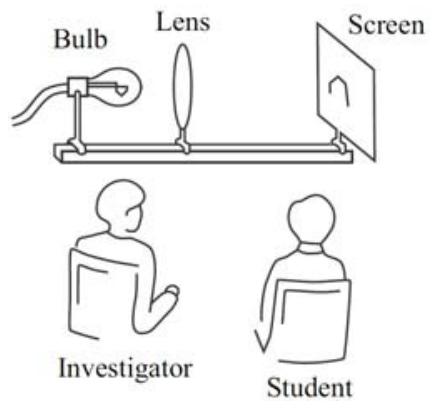

Figure 1. Individual demonstration interview.

The students were first asked what change would occur on the screen if the lens were removed. About half said that it would be right side up, apparently not recognizing the necessity of the lens to form the image. They seemed to think that the function of the lens was to invert the image. The students were also asked what would happen if the top half of the lens were blocked. Only about onethird seemed to recognize that even a very small part of a lens is sufficient to produce an image. The most popular response was "half a lens, half an image." When asked to draw a ray diagram, many of the students did not seem to understand that tracing the principal rays through a lens is an algorithm, or rule, for locating the image. The special rays are sufficient, but not necessary. In another task, the students were asked if anything would change on the screen if it were moved toward the lens. Fewer than half recognized that, if the screen were moved very far in either direction, no image would appear.

A multiple-choice version of these questions that was given to many students led to a similar outcome. The results were essentially the same whether or not students were enrolled in the accompanying laboratory course. Even more disturbing was the fact that the results did not depend on whether or not the students had studied this material in high school or whether or not they had just completed this topic in the university course in which they were enrolled.

Research Task: Geometric image formed by an aperture. An investigation conducted several years later indicated that the difficulties that the introductory students had with the questions above were much more fundamental than a lack of understanding of the function of a lens (Wosilait et al, 1998). To determine whether they could apply a correct ray model in a situation not involving a lens, we asked more than 2000 students in the introductory calculus-based course to sketch the images produced on a screen when light from a single point source, two point sources, and a line source passes through a small triangular hole $(\sim 1 \mathrm{~cm})$ in a mask. This question was also given to more than 100 graduate students in our Physics Department and to many pre-university teachers. 


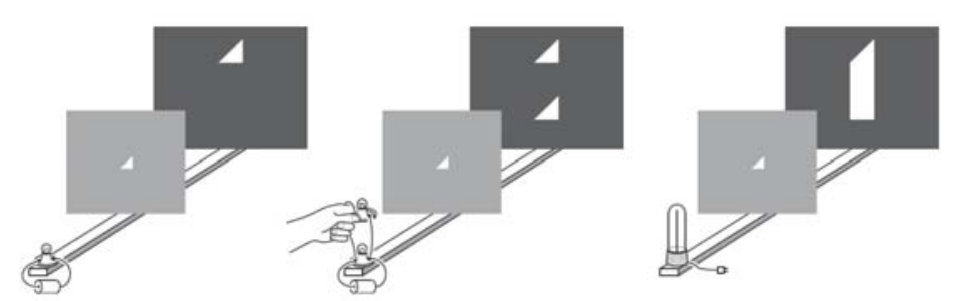

Figure 2. Pretest (with correct responses).

As in the tasks based on the converging lens shown in Fig. 1, memorized formulas do not help. A correct response depends on the ability to apply a correct ray model. To draw an appropriate diagram in the third case, students could treat the line source as a series of point sources, each of which produces a triangular image on the screen. Since the points are closely spaced, the images overlap and produce a vertical rectangle terminated at the top by a triangle.

Although the amount of prior instruction varied, the results did not. The percentages of correct responses for the three light sources by the introductory students were $90 \%, 60 \%$, and $20 \%$, respectively. The performance of the elementary and secondary school teachers who were asked this question was similar. The graduate students did better on the first two questions, but only about $65 \%$ gave a correct (or nearly correct) answer for the line source. The most common incorrect response in all three groups was that the image would be a stretched triangle.

\section{Tutorials: an instructional approach for addressing student difficulties}

The motivation for developing Tutorials in Introductory Physics was our desire to provide for introductory students at least some of the intellectual experience that $P b I$ provides for teachers. We were faced with the challenge of trying to secure the mental engagement of students in large classes. Our one-year introductory calculus-based physics course has about 1000 students. There is the additional complication that all three academic quarters of this course (which at that time ranged from mechanics to physical optics) are taught concurrently. A great deal of material is covered very rapidly in lectures attended by 100-200 students. There are eight lecture sections with eight different instructors. Faculty rotate through the course on a cycle that varies from one academic quarter to three years. A weekly three-hour laboratory is part of the course. There are 45 laboratory sections. Although our faculty are conscientious in fulfilling their instructional responsibilities, the Department is strongly research-oriented. It would be unrealistic to ask the faculty to spend more time in teaching. We needed a system that would be practical, flexible, and sustainable. Our response to the challenge was the development of Tutorials in Introductory Physics, which is the core of the tutorial system that we have implemented in the course. We chose the word "tutorial" to distinguish the small-group sessions that are based on our curriculum from the recitation, discussion, or quiz sections traditionally associated with small-group science instruction in the U.S. The usual way of conducting such small-group sessions in physics is for a faculty member, or Teaching Assistant (TA), to work problems for the students, ask them to solve problems, or respond to questions (often with a minilecture). The tutorials that we have developed are very different in purpose and in structure.

\section{Nature of the tutorials}


The tutorials respond to the research questions: Is the standard presentation in textbook and lecture adequate to develop a functional understanding? If not, what can be done? The tutorials are intended to supplement, not replace, instruction by lecture, laboratory, and textbook. They are designed to target critical ideas and skills that are known through research and teaching experience to present difficulty to students. The emphasis in the tutorials is on constructing concepts, on developing reasoning skills, and on relating the formalism of physics to the real world, not on transmitting information and solving standard problems.

\section{Description of the tutorial system}

The tutorial system provides the context in which much of our research and curriculum development take place. Each tutorial sequence consists of a pretest, worksheet, homework assignment, and one or more post-test questions. The sequence begins with a 10-minute pretest (so named because it precedes the tutorial, although the material may have already been covered in lecture). The pretests, which are administered either in lecture or on the internet, have several purposes: to alert students to what they need to know and be able to do, to set the stage for the associated tutorial, and to inform the course lecturers and tutorial instructors about the intellectual state of their students. Most pretests have been designed to elicit known difficulties. During the weekly 50-minute tutorial sessions, about 20-24 students work collaboratively in groups of 3 or 4 . The structure is provided by tutorial worksheets that have been designed to help students confront and resolve specific difficulties. The worksheets contain questions that try to break the reasoning process into steps of just the right size for students to become actively involved. If the steps are too small, little thinking may be necessary. If the steps are too large, the students may become lost unless an instructor is by their side. The tutorial homework assignments help students reinforce and extend what they have learned.

To secure the level of intellectual commitment necessary to bring about a significant conceptual change, the tutorials employ a variety of instructional strategies. One that has proved particularly effective can be summarized as a sequence of steps: elicit, confront, and resolve. The first step is to elicit a known difficulty by contriving a situation in which students are likely to make an error that exposes that particular difficulty. They then need to recognize the source of the error. Many students are willing to tolerate inconsistency. It is the responsibility of the instructor to insist that students confront and resolve underlying difficulties. If these are sufficiently serious and not addressed, they may remain latent and arise in other contexts. Tutorial homework provides additional practice that helps reinforce the ideas developed in the tutorials. The students are given the opportunity to apply the relevant concepts in related but different contexts, to reflect, and to generalize.

At least one-fourth of every course examination requires qualitative reasoning and verbal explanations. The students quickly learn that attending lectures and reading the textbook are not sufficient preparation. Questions based on the tutorials are included on every course examination and serve as post-tests.

\section{Development of a tutorial: Light and shadow}

We developed the tutorial Light and Shadow to help students apply two basic principles from geometrical optics: light travels in straight lines and light rays from every point on an object travel outward in all directions. The tutorial sequence begins with a pretest based on the apparatus shown in Fig.2 and described earlier. Guided by the results from the pretest, we designed a tutorial Light and Shadow (McDermott and Shaffer, 2002). It begins by asking students to predict the images formed by point and line sources with apertures of various sizes and shapes. After the students have made predictions and explained their reasoning to one another, they observe what actually happens and try to resolve any discrepancies with their predictions. They are then asked to predict and explain updown and left-right inversions of images produced by asymmetric sources. These and other exercises 
help students recognize that the size and shape of the source, the size and shape of the aperture, and the distances involved can all affect the image. The students note that whether a light source can be treated as a point, a line, or an extended source depends on a variety of factors.

Systematic monitoring in the classroom helped improve the tutorial. One exercise was added that has had a pronounced effect. The students are asked to predict what they would see on the screen when a large frosted bulb is used as the light source. Many predict a triangular image and are surprised to see the inverted image of the bulb on the screen. They eventually realize that the entire bulb can be considered as a collection of point sources. Superposition of the images from the continuum of point sources produces an image that closely resembles the shape of the extended source, but is affected by the shape of the aperture at the edges, where fewer images of the aperture overlap with one another.

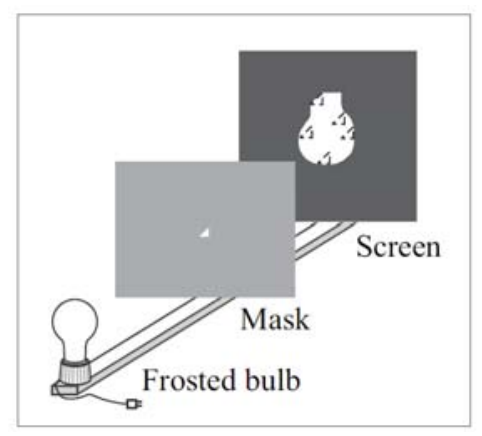

Figure 3. Image of frosted bulb resulting from superposition of images of triangular hole.

\section{Assessment of the tutorial: Light and shadow}

Throughout development of the tutorial, assessment played a critical role. Post-test questions were administered on different course examinations to about 360 students who had worked through the tutorial. Since the post-tests are always administered on course examinations, they provide accurate feedback on whether students have understood what they were expected to learn from the tutorial. Several of the post-test questions (with correct responses) are shown in Fig. 4.

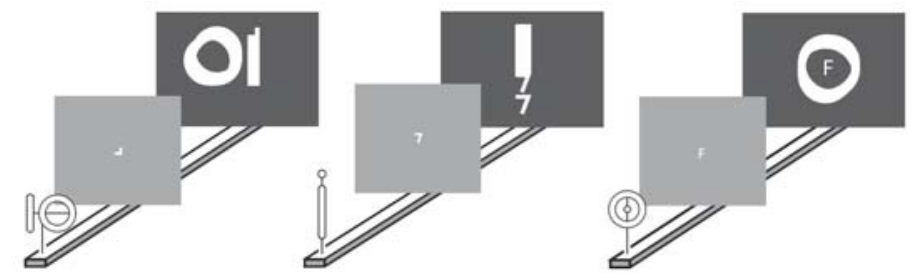

Figure 4. Post-tests (with correct responses)

We counted as correct, or nearly correct, all responses that indicated either that the extended source was composed of point sources or that the image was basically the same shape as the source. About $80 \%$ of the introductory students gave correct (or nearly correct) answers on the post-tests, as did about $85 \%$ of the prospective secondary school teachers who had worked through similar material in $\mathrm{PbI}$ (McDermott et al, 2006). This result represented a substantial increase over the $20 \%$ on the pretest. Only $10 \%$ drew images the same shape as the aperture, in sharp contrast to the $70 \%$ who made this error on the pretest.

Pretest scores on the long-filament bulb from 110 TA's and post-docs showed that about $65 \%$ gave a correct, or nearly correct, response. About 30\% drew a triangular image. Comparison of their average 
scores with the post-test performance of the introductory students indicates that the undergraduates achieved a better functional understanding of this material than the graduate students initially had.

About 200 participants in our faculty development workshops have taken a pretest slightly more difficult than the one in Fig. 2. About 45\% have given a correct, or nearly correct, response for the long-filament bulb. A few participants in our faculty development workshops have predicted a triangular image. After working through the tutorial, some have remembered that during a solar eclipse they had seen multiple images on the ground of the partially eclipsed sun beneath a canopy of leaves.

We have found, both from our own experience and that of other faculty, that even those with a Ph.D. in physics can deepen their understanding by doing simple experiments and carefully reflecting on the outcome. Our experience has been that voluntary time spent in working through a tutorial often varies inversely with the background one has had in physics. Faculty, post-docs, and graduate students frequently spend more time on a tutorial than undergraduates.

\section{Requirements for successful implementation of the tutorials}

There are two very important conditions that must be met for successful implementation of the tutorials in an introductory physics course. Students must understand the importance of the type of reasoning that they are expected to learn and the tutorial instructors must recognize that teaching by questioning demands more careful preparation than teaching by telling.

Importance of examinations. If students are to be expected to do the reasoning required for the development and application of concepts, they must be held accountable for developing the required skills. The most effective (and probably the only) way is to insist that they explain their reasoning on course examinations. Requiring students to give explanations challenges a common tendency to think of physics as a collection of facts and formulas. The tutorials would not have been nearly as successful in improving student learning at UW if post-tests had not been part of all examinations and, hence, a significant component of the course grade.

Preparation of tutorial instructors. The tutorial system would not work without ongoing preparation of the tutorial instructors in both the subject matter and the instructional method. Most of us teach as we were taught. It is unrealistic to expect peer instructors, graduate TA's, or faculty to be able, without preparation, to teach by questioning in a way that promotes development of reasoning skills. The instructors who conduct the tutorial sessions are Teaching Assistants (TAs). Most are physics Ph.D. students. Our research has shown that they often have the same conceptual and reasoning difficulties as undergraduates. However, knowing the answer to the questions on the tutorial worksheet is not adequate. It is much easier to give answers and explain why they are correct than it is to formulate questions that guide students through the reasoning involved. Instructors in the tutorials need to learn how to teach by questioning (rather than by telling). They develop this skill in a required weekly Graduate Teaching Seminar, which is conducted on the same material and in the same manner as the tutorial instructors are expected to teach. During the Seminar, the TAs examine responses given by students, try to identify their difficulties, and attempt to determine whether they have used correct reasoning.

Because the TAs take the same pretests as the introductory students, we have a measure of their level of understanding. Although they can generally solve the end-of-chapter problems, they often do not have a sufficiently strong command of the material for the type of teaching by questioning that the tutorials require. We have found that advanced study does not necessarily lead to a deeper understanding of introductory physics. We consider a tutorial successful when the performance of the 
introductory students on post-tests matches (or surpasses) that of the Graduate Teaching Assistants (TAs) on the corresponding pretests.

\section{Efforts to substitute standard lecture-based instruction for tutorials}

Some instructors have tried to adapt the instructional strategies in the tutorials to a lecture format. It is interesting to examine what happened when two professors, who were teaching different lecture sections of the same calculus-based course, decided that having students work through the tutorial Light and shadow would take too much class time. One of the classes consisted of honors students; the other was a regular section of the same course. Both professors were experienced and popular lecturers who liked our tutorials and were aware of the conceptual difficulties that they have been designed to address. Instead of asking their students to work through the Light and shadow tutorial, they showed demonstrations during their lectures and explained the ideas. They required their students to work through modified versions of the tutorial as homework.

On the pretest, the 30 students in the honors section had an average score of $25 \%$, which was slightly higher than the average of $20 \%$ achieved by the more than 1000 students in the regular sections. On the post-test, however, the performance of students in the two sections taught by these professors was well below the average of all of the students who had worked through the tutorial. After doing the homework, the students in the honors section had an average score of $45 \%$, while those in the other section had an average score of $35 \%$. Moreover, the prevalence of the mistaken idea that the image mimics the hole in the mask was still quite high $(40 \%$ in the honors section and $35 \%$ in the regular section). These percentages exceeded the $10 \%$ who made this error in all of the sections in which the students had worked through the tutorial.

Apparently, teaching by telling is not nearly as effective as teaching by questioning. It is insufficient for the instructor to give clear explanations, show demonstrations, assign problems for homework, and provide solutions. Even if the instructor is well aware of student difficulties, he or she cannot do the students' thinking for them. Their active mental engagement is necessary. For most students, intellectual involvement at a sufficiently deep level does not take place unless they are required to work step-by-step through instructional materials that have been carefully designed and tested.

The tutorials are designed to help make physics meaningful for students, to help them develop scientific reasoning skills, and to help them recognize that how we know is as important as what we know. Although the implementation of tutorials means that less time is devoted to solving standard textbook problems, success in solving quantitative problems is not diminished and is often increased. Placing a greater emphasis on qualitative reasoning thus raises standards. The tutorial system has proved to be practical, flexible, and sustainable in our research-oriented physics department. We have also been developing tutorials on more difficult material (e.g., special relativity and quantum mechanics) and have observed that students in more advanced courses welcome the opportunity provided by tutorials to deepen their conceptual understanding. We have found, that the same instructional approach also works well for these students.

\section{Need for special courses for the preparation of teachers}

Our group has conducted many studies that have included elementary and secondary school teachers. We have identified many of the same intellectual problems in this population as in others (McDermott et al, 2006). However, teachers must have an especially sound command of the material. They not only need to overcome their own conceptual and reasoning difficulties, but must also learn to recognize and be able to address the difficulties that their students are likely to encounter. 
Moreover, if they are to be expected to teach science as a process of inquiry, rather than as a compendium of facts to be memorized, teachers should have some direct experience in this way of learning. They should be given the opportunity to learn (or relearn) the physics that they are expected to teach in a manner that is consistent with how they are expected to teach. The time usually allotted for tutorials ( one hour/week) is insufficient for this purpose. Special courses in physics are needed in which the development of a deep functional understanding of basic concepts is emphasized and instructional methods are taught by example (McDermott, 1974, 1990, 2006; McDermott et al, 2006).

Physics by Inquiry, which is more thorough than the tutorials, has been developed for such courses and has also been used with other populations. $\mathrm{PbI}$ is a self-contained, laboratory-based curriculum that we began to develop in the 1970s. It is the "ancestor" of the tutorials and is designed to help elementary, middle, and high school teachers develop the competence and confidence needed to be able to teach physics and physical science effectively. $\mathrm{PbI}$ provides the opportunity for teachers to learn (or relearn) science in a way consistent with how they are expected to teach. Two broad principles characterize this curriculum:

- Concepts, reasoning ability, and representational skills are developed together within a coherent body of subject matter.

- Physics is taught as a process of inquiry, not as an inert body of information.

Physics by Inquiry consists of a set of modules, all of which require active participation by the learner. In all of the modules, observations and simple experiments provide the basis on which students construct physical concepts and develop analytical reasoning skills. The development of concepts begins with observations made by students. Starting with a few plausible assumptions, the curriculum guides students through a chain of reasoning that enables them to construct conceptual models that they can apply to predict and explain the behavior of some common physical phenomena (e.g., bulb brightness in electric circuits, geometric images formed by apertures, and phases of the moon). The topics have been chosen to provide K-12 teachers with the experience needed to be able to teach science in the K-12 grades by inquiry, rather than by lecture.

The preparation of teachers should emphasize intellectual development rather than wide coverage of many topics. Courses that provide an overview of contemporary physics can be inspiring, but they should not be considered a substitute for helping teachers develop a deep understanding of the basic concepts that have led to our present knowledge. The claim that one knows how to prepare teachers to teach their students about recent advances in physics should be carefully examined. Such courses may be inspiring for teachers but they are not adequate for preparing them to teach that material. The criterion that should be used in determining the validity of a claim that a teaching sequence has been successful with teachers (or students) should include information about the nature and depth of the assessment questions asked and the form in which the questions were asked. Such considerations are particularly important in determining whether teachers have been sufficiently well prepared to teach what they have been taught.

\section{Research-based and research-validated curriculum}

Until fairly recently our group was accustomed to referring to Physics by Inquiry and Tutorials in Introductory Physics as research-based. However, current overuse use of that term is creating a problem. Almost every new text today is promoted as research-based. What seems to be lacking is the recognition that research-based is not equivalent to research-validated. Just because one is aware of what students cannot do does not mean that one knows what to do about the situation. Unless instructional strategies have been shown to work reproducibly, merely stating that a text is research-based is not a strong recommendation. A higher standard is needed - one that is based on evidence, not on expectation or opinion. Both $\mathrm{PbI}$ and the tutorials are research-based and research-validated 


\section{Generalizations about learning and teaching}

Several generalizations about learning and teaching that we have inferred from our research have guided the development of both Physics by Inquiry and Tutorials in Introductory Physics. (McDermott, 2001). Below, some of our generalizations about learning are paired with italicized generalizations about teaching.

- Facility in solving standard quantitative problems is not an adequate criterion for functional understanding. Questions that require qualitative reasoning and verbal explanations are essential to assess student learning. The use of such questions is also an effective strategy to help students learn.

- A coherent conceptual framework is not typically an outcome of traditional instruction. Students need to go through the process of constructing models and applying them to predict and explain real-world phenomena.

- Certain conceptual difficulties are not overcome by traditional instruction. (Advanced study may not increase student understanding of basic concepts.) Persistent conceptual difficulties must be explicitly addressed.

- Connections among concepts, formal representations (diagrams, graphs, etc.) and the real world are often lacking after traditional instruction. Students need repeated practice in interpreting physics formalism and relating it to the real world.

- Growth in reasoning ability does not usually result from traditional instruction. Scientific reasoning skills must be expressly cultivated.

- Teaching by telling is an ineffective mode for most students. Students must be intellectually active to develop a functional understanding.

\section{Conclusion}

There is a need in physics and other sciences for research on the intellectual development of students as they progress through a body of material. We attribute the effectiveness of Physics by Inquiry and Tutorials in Introductory Physics to the detailed knowledge of student difficulties that informed their development. The depth of understanding that is required for such research is found only among specialists in a field and therefore must be conducted by science faculty in science departments. When reported at professional meetings and in journal articles, the results provide a rich resource for all who teach. Thus, cumulative improvement in the effectiveness of instruction becomes possible.

\section{Acknowledgements}

The accomplishments of the Physics Education Group are the result of close collaboration among all of its members. In addition to Paula Heron and Peter Shaffer, both of whom contributed to the research described in this paper, other senior members of the group are Donna Messina and MacKenzie Stetzer. Karen Wosilait was the primary author of the tutorial on geometrical optics that has served as the context for much of the discussion. The cooperation of colleagues at the University of Washington and at our pilot sites is greatly appreciated. Support by the U.S. National Science Foundation has made our work possible.

\section{References}


Goldberg F. M. and McDermott L. C. (1987). An investigation of student understanding of the real image formed by a converging lens or concave mirror. American Journal of Physics, 55(2), 108-119.

Heron, P. R. L. and McDermott, L. C. (1998). Bridging the gap between teaching and learning in geometrical optics: The role of research. Optics and Photonics News, 9(9), 30-36.

McDermott L. C. (1974). Combined physics course for future elementary and secondary school teachers. American Journal of Physics, 42(8), 668-676.

McDermott L. C. (1990). A perspective on teacher preparation in physics and other sciences: The need for special science courses for teachers. American Journal of Physics, 58(8), 734-742.

McDermott L. C. and the Physics Education Group at the University of Washington (1996). Physics by Inquiry. New York, NY: Wiley.

McDermott L. C. (2001). Oersted Medal Lecture 2001: "Physics Education Research-The Key to Student Learning. American Journal of Physics, 69(11), 1127-1137.

McDermott L. C., Shaffer P. S., and the Physics Education Group at the University of Washington (2002). Tutorials in Introductory Physics, First edition. Upper Saddle River, NJ: Prentice Hall.

McDermott L. C. (2006). Invited Editorial: Preparing K-12 teachers in physics: Insights from history, experience, and research. American Journal of Physics, 74(9), 758-762.

McDermott L.C., Heron P.R.L, Shaffer P. S., and Stetzer, M.R. (2006). Improving the preparation of K-12 teachers through physics education research. American Journal of Physics, 74(9), 763-767.

Wosilait K., Heron P. R.L., Shaffer P. S., and McDermott L. C. (1998). Development and assessment of a research-based tutorial on light and shadow. American Journal of Physics, 66(10), 906-913 